\title{
La qualité en formation à distance : une question de points de vue?
}

\section{Quality in Distance Learning: A Question of Perspectives?}

\section{La calidad en la enseñanza a distancia: ¿una cuestión de perspectivas?}

Cathia Papi, professeure

Université Téluq, Québec

Cathia.Papi@teluq.ca

Marie-Hélène Hébert, professeure

Université Téluq, Québec

marie-helene.hebert@teluq.ca

Avec cet article, nous enrichissons le débat sur ce qui est entendu par « qualité » en formation à distance, en partant du présupposé qu'il n'existe pas une conception unanime et facilement contrôlable de la qualité. Pour ce, nous avons mené une enquête auprès de 12 acteurs d'une université à distance jouant différents rôles touchant à la conception des cours, leur médiatisation et leur diffusion, à l'encadrement et l'évaluation des étudiants et finalement, au travail de coordination et de gestion académiques. Nos résultats montrent que la définition de la qualité de chaque acteur dépend en grande partie du rôle qu'il joue dans la formation à distance, mais que certaines conceptions sont cependant partagées.

Mots-clés : qualité, formation à distance, conceptions des acteurs, enseignement supérieur 
With this article, we enrich the debate on what is meant by "quality" in distance education, based on the assumption that there is no unanimous and easily controllable conception of quality. To this end, we conducted a survey of 12 actors in a distance university who play different roles in course design, media coverage and dissemination, student supervision and evaluation, and finally, academic coordination and management. Our results show that each actor's definition of quality depends mainly on their role in distance education, but that certain conceptions are nevertheless shared.

Keywords: quality, distance education, actors' conceptions, higher education

\section{RESUMEN}

Con este artículo enriquecemos el debate sobre lo que se entiende por "calidad" en la educación a distancia, partiendo del supuesto de que no existe una concepción unánime y fácilmente controlable de la calidad. Por eso, realizamos una encuesta con 12 actores de una universidad a distancia que desempeñan diferentes papeles en el diseño de los cursos, la coberturasu mediatización y la difusión en los medios de comunicación, la supervisión y evaluación de los estudiantes y, por último, la coordinación y la gestión académica. Nuestros resultados muestran que la definición de la calidad de cada actor depende en gran medida del papel que desempeñandesempeña en la educación a distancia, pero que, sin embargo, se comparten ciertas concepciones.

Palabras clave: calidad, educación a distancia, concepciones de los actores, educación superior

\section{Introduction}

Alors que la question de la qualité en formation à distance (FAD) est apparue dès son essor sous forme de formation en ligne avec la diffusion d'internet à la fin du $20^{\text {ème }}$ siècle, force est de constater que différentes approches visant le contrôle de la qualité coexistent. II est notamment possible de citer celles portant sur les normes techniques assurant l'interopérabilité des ressources; celles ayant trait au matériel ou contenu d'apprentissage; celles centrées sur l'encadrement offert aux étudiants et enfin, celles prenant en considération la persévérance, les résultats ou la satisfaction des étudiants comme gage de qualité (Pham et al., 2019; Esfijani, 2018; Savarieau et Daguet, 2013; Depover, Komis et Karsenti, 2012; De Lièvre, 2005; Ehlers, 2004). Si tous ces éléments peuvent être pris en compte comme des critères de qualité, aucun ne semble garantir à lui seul la bonne qualité d'un dispositif de FAD. D'où la nécessité de conjuguer plusieurs indicateurs comme le propose, par exemple, le SLOAN consortium (Bourne et Moore, 2003) au début des années 2000 en considérant la qualité comme étant à la jonction entre l'efficacité pédagogique, la satisfaction des étudiants, la satisfaction du corps professoral, le rapport coût-efficacité et la facilité d'accès aux contenus et services de la formation. Cependant, pas plus cette conception de la qualité qu'une autre ne s'est généralisée. Ainsi, ayant réalisé une analyse systématique de la littérature en anglais dans le domaine entre 2000 et 2017, Esfijani (2018) fait ressortir que les travaux relatifs à la qualité de la FAD sont nombreux et qu'aucun consensus sur le sens, l'approche et la mesure de la qualité n'existe. Dès 2004, Hew et ses collaborateurs tentent de faire des regroupements en mettant en avant 
que trois niveaux de la qualité de la FAD sont pris en compte dans la littérature, à savoir le niveau macro de la qualité des programmes, celui méso de la qualité des cours et celui micro de l'apprentissage des étudiants. Selon les définitions de la qualité et niveaux considérés, les modèles et outils de mesure proposés varient de telle sorte qu'Esfijani (2018) souligne le manque d'un modèle holistique et remarque également que, tandis que les conceptions de la qualité prennent souvent en compte les étudiants, le corps enseignant ou les administrateurs, celles prenant en considération d'autres acteurs tels que les concepteurs de la formation et les employés de soutien sont rares. Or, la participation de chaque acteur, tant au niveau de la production que de l'encadrement, semble nécessaire pour parvenir à une formation de qualité. Dès lors, quelles sont les conceptions de la qualité des différents acteurs nécessaires à la création et à la diffusion d'une FAD? Sont-elles différentes selon les acteurs ou peut-on donner une définition ou un ensemble de principes gages de qualité? Entre représentation générale et représentation individuelle de ce qu'est la qualité en FAD, nous faisons l'hypothèse que la définition de la qualité de chaque acteur dépend du rôle qu'il joue dans la FAD.

\section{Objectif : participer au débat sur ce qu'est la qualité en FAD}

Pour enrichir le débat sur ce qui est entendu par « qualité » en FAD, nous proposons de nous intéresser aux points de vue de différents acteurs employés par une université à distance afin de mettre en avant leurs définitions de la qualité et de les croiser pour ouvrir la discussion en nous demandant si, à l'instar de la complémentarité de leurs activités dans le cadre de la FAD, leurs conceptions de la qualité se complèteraient. Ce qui ouvrira la voie vers une approche plus complète concernant la production et la diffusion de FAD de qualité. Ce faisant, nous proposons une réflexion originale, qui vise à mettre quelque peu en lumière un pan d'ombre de la recherche sur la qualité. S'il s'agit avant tout d'une discussion qui ne prétend pas être une recherche au sens propre du terme, celle-ci s'appuie cependant sur une enquête à petite échelle et une analyse menée avec rigueur.

\section{Méthodologie : une enquête à l'Université TÉLUQ}

Comme terrain pour l'enquête, nous avons fait le choix de l'Université TÉLUQ, une institution d'enseignement et de recherche québécoise qui œuvre en FAD depuis 1972. Offrant une programmation de plus de 400 cours et 125 programmes d'études, elle accueille près de 20000 étudiants par année. Pour accomplir sa mission de production et de diffusion du savoir à distance, elle compte sur le soutien de plus de 500 acteurs qui y mènent des activités complémentaires, mais essentielles. Douze d'entre eux ont servi d'échantillon à l'enquête : deux professeurs, une spécialiste en sciences de l'éducation, une spécialiste en design d'édition pédagogique, une commis à l'édition assistée par ordinateur, une technicienne en informatique, une spécialiste en communication écrite, un responsable des droits d'auteur et de l'approvisionnement des volumes utilisés dans les cours, deux chargés d'encadrement d'étudiants, une coordonnatrice à l'encadrement et, enfin, une agente de gestion des études.

Pour faire émerger leurs conceptions de la qualité en $F A D$, cinq questions à développement long leur ont été administrées par courriel en décembre 2019 :

1. À quoi correspond, pour toi, la qualité en formation à distance?

2. Dans ta pratique, comment la mets-tu en œuvre?

3. Quel serait un exemple de bonne qualité dans ton domaine? 
4. Quel serait un exemple de non-qualité ou de manque de qualité dans ton domaine?

5. Que faire pour améliorer la qualité dans cet exemple?

Les réponses des 12 acteurs ont été lues à plusieurs reprises, puis les éléments les plus significatifs de leur discours sur la qualité ont été regroupés et catégorisés selon les différents rôles qu'ils jouent dans la FAD : la conception des cours, leur médiatisation et leur diffusion, l'encadrement et l'évaluation des étudiants et finalement, l'essentiel travail de coordination ou de gestion académique qui se déploie en filigrane, de l'admission de l'étudiant dans son programme d'études à sa diplomation.

\section{Résultats : une diversité de points de vue}

Nous présenterons tout d'abord les résultats concernant les définitions de la qualité selon les rôles joués par les acteurs enquêtés dans la FAD. Certains en jouant plusieurs, tels que les professeurs participant aussi bien à la conception des cours qu'à l'encadrement; par exemple, nous avons compilé pour chaque rôle les propos d'au moins trois des 12 acteurs. Autrement dit, au-delà des exemples cités propres à un enquêté en particulier, les propos de synthèse renvoient à plusieurs témoignages. Nous proposons d'en faire le tour dans l'ordre habituel allant de la conception des cours à l'évaluation des travaux des étudiants.

\section{Conception}

Lors de la phase de conception, la qualité est vue à deux niveaux. L'un d'eux est centré sur le contenu du cours qui doit être à jour, varié (diversité de contenu) et dont les textes écrits doivent être rédigés dans un « français impeccable » exempt d'anglicisme et facilement lisible (privilégier les phrases courtes, prêter attention à l'uniformité des termes). L'autre niveau concerne davantage la conception pédagogique en mettant en relief une recherche de cohérence entre les objectifs pédagogiques et le contenu (ressources, activités) des cours en vue de favoriser la réussite des étudiants. Les technologies sont ainsi considérées comme des moyens au service de la pédagogie. Elles doivent être conviviales et attrayantes et favoriser l'accessibilité des cours en contribuant notamment à l'application de la conception universelle de l'apprentissage (CUA). Afin d'atteindre la qualité souhaitée, trois éléments semblent pris en compte lors de la phase de conception : « mon travail de design est guidé, d'un côté, par les orientations, les principes et les recommandations issus de la recherche dans le domaine et, d'un autre côté, par les bonnes pratiques d'exploitation des technologies. Pour moi, c'est aussi très important de toujours penser à la perspective de l'étudiant, qu'est-ce qui pourrait faciliter son apprentissage? Qu'est-ce qui pourrait renforcer sa motivation? Qu'est-ce qui pourrait favoriser le développement des habiletés et des compétences visées par le dispositif de formation? Avoir ces questionnements toujours à l'esprit m'aide à mettre en place la structure la plus adaptée aux besoins des étudiants. "

\section{Médiatisation}

Pour ce qui est de la médiatisation, la qualité est comprise en termes d'accessibilité du contenu. Autrement dit, il s'agit de faire en sorte que la navigation dans le site Web du cours soit aisée, que les contenus soient facilement repérables, adaptables aux situations et technologies des étudiants. Ces dernières sont vues comme un atout dont il convient de tirer profit pour faciliter l'apprentissage de l'étudiant. Pour atteindre la qualité visée, il est précisé qu'« il est important de rencontrer tous les membres de l'équipe de production de la formation dès le début du projet. Un suivi avec une bonne communication et des échanges réguliers favorise l'harmonie du travail. » L'utilisation de technologies récentes et la prise en compte de l'ergonomie favorisant l'accessibilité sont également mises en avant. Enfin, il ressort que les apprenants doivent être au cœur de chacune des décisions prises, à tous les niveaux, " pour leur offrir la meilleure expérience possible. » 


\section{Diffusion}

Au niveau de la diffusion, la qualité fait référence à la correction de la langue, à la lisibilité des textes et des autres ressources et à l'attention portée aux droits d'auteurs en cherchant à concilier le respect du droit tout en ne faisant l'acquisition que du nécessaire, mais en s'assurant aussi d'avoir tout ce dont l'étudiant a besoin, notamment en termes d'ouvrages. Ainsi, un enquêté explique : « La qualité passe donc d'abord par des vérifications auprès des maisons d'édition afin de nous assurer que leurs titres ne tomberont pas en arrêt de commercialisation à brève échéance ou qu'une nouvelle édition ne serait pas en préparation. Imaginez l'impact si un cours était monté en fonction d'un volume qui n'existera plus : une perte importante de temps et d'argent à coup sûr!»

\section{Coordination}

En ce qui concerne les activités de coordination ou de gestion académique, la qualité renvoie au soutien apporté à l'étudiant au niveau de son inscription, de son cheminement, de la maîtrise technologique et de l'accès aux ressources et services. Elle se définit plus particulièrement par la rigueur des réponses et l'ensemble des services offerts aux étudiants, tuteurs, chargés d'encadrement ou professeurs. II s'agit dans ce cadre de bien analyser les dossiers, les programmes et règlements avant de répondre. Ainsi, pour atteindre la qualité envisagée, une enquêtée précise : « J'essaie de répondre le plus rapidement possible, tout en étant claire dans mes réponses, en vérifiant l'exactitude des informations, en consultant les personnes concernées pour certaines décisions, en s'assurant d'avoir une concertation si possible et ce, en fonction des limites de mon champ de responsabilité. »

\section{Encadrement}

Le rôle d'encadrement est perçu comme fort important par ceux qui l'exercent. En effet, ces derniers considèrent qu'un bon encadrement peut pallier une mauvaise conception des cours. En ce sens, la qualité de l'encadrement joue un rôle non négligeable concernant la persévérance et la réussite des étudiants, un encadrement de piètre qualité pouvant quant à lui conduire à l'insatisfaction, à la procrastination, voire à l'abandon. Un encadrement de qualité est caractérisé par la diligence, la politesse, la maitrise du cours et des plans de soutien à l'apprentissage. II s'agit ainsi de communiquer avec l'apprenant, d'être accessible pour lui et chercher à le comprendre et assurer une proximité, "le rejoindre dans son monde », pour personnaliser la démarche d'apprentissage, le valoriser et le guider dans ses apprentissages et dans le développement de son autonomie. Pour atteindre la qualité, un enquêté indique : « un travail d'équipe est je crois nécessaire, par tous les intervenants impliqués. Une sensibilisation à la FAD (atelier, formation, capsules...), facilitant l'engagement plein et entier de l'étudiant, est certainement une piste à considérer. »

\section{Évaluation}

En termes d'évaluation des apprentissages, la qualité est perçue comme consistant à fournir de nombreuses rétroactions pour permettre à l'étudiant de progresser, à donner un barème expliquant où des points ont été perdus, à vérifier le respect des consignes et normes. Par ailleurs, comme en ce qui concerne l'encadrement, le fait de répondre rapidement est considéré comme une caractéristique de la qualité, une preuve de respect de l'étudiant. Mettre en œuvre une évaluation de qualité implique ainsi, pour l'un des enquêtés, de « mettre autant de commentaires que possible, mais sans aller dans le ridicule 
de commenter chaque ligne d'un travail. II faut joindre un barème, un tableau pour dire où les points ont été perdus. II faut ajouter des commentaires à ce tableau, à ce barème pour guider l'étudiant, mais pas trop. II ne faut pas que ce barème soit une vérité, mais doit donner une bonne idée de pourquoi il a perdu des points. "

\section{Discussion : du débat au consensus?}

En menant cette enquête, nous nous attendions à voir chez les acteurs des conceptions très individuelles et contrastées de la notion de qualité et à pouvoir ouvrir un réel débat confrontant différents points de vue. Or, à la première lecture de leurs réponses, nous avons compris que tel n'était pas le cas, mais que pour autant des différences de références apparaissaient selon le rôle joué dans le processus de production ou d'accompagnement de la formation. D'où le choix de faire une analyse par domaine d'exercice des acteurs dans la FAD comme présenté ci-dessus. En effet, bien que pour chaque domaine des acteurs aux statuts différents aient été enquêtés, force est de constater que les idées avancées par différents acteurs jouant un rôle dans un des domaines cités ont pu être synthétisées très aisément tant elles étaient proches. D'ailleurs, il est intéressant de constater que des éléments reviennent dans différents domaines. Ainsi, la qualité en FAD semble principalement se définir au niveau de la présentation du contenu (tant au niveau linguistique que de la variété des médias), de la cohérence du parcours pédagogique proposé et de l'accompagnement apporté à l'étudiant allant de l'inscription à l'évaluation. Pour parvenir à la qualité, la plupart des acteurs soulignent, indépendamment de leur rôle, l'importance de faire son travail en gardant en tête ce qui est le plus favorable aux étudiants, l'interdépendance du travail des uns et des autres, le respect des différentes étapes dans la création de FAD et enfin, la rigueur nécessaire à l'ensemble des activités allant de la conception à l'évaluation de FAD, car « la qualité, c'est l'affaire de tous! ». Alors que les étudiants jouent ainsi un rôle central en tant que destinataires de la formation pour lesquels la recherche de qualité est forte dans les différents domaines, il serait ainsi intéressant de confronter leurs points de vue à ceux recueillis dans le cadre de cette enquête. Si nous ne nous y sommes ici pas intéressées, il convient de préciser que leurs opinions concernant la qualité des cours et de l'encadrement reçus sont recueillies dans un processus d'évaluation institutionnelle qui pourra faire l'objet d'un autre article de médiations \& médiatisations.

\section{Liste de références}

Bourne, J. et Moore, J. C. (dir). (2003). Elements of quality online education: Practice and direction. Needham, MA: SLOAN Consortium.

De Lièvre, B. (2005). La qualité du tutorat : la complémentarité de la rigueur et de la diversité. In $2^{\text {ième }}$ Colloque Euro Méditerranéen et Africain d'Approfondissement de la FORmation A Distance (CEMAFORAD), Université Abderrahmane Mira, Béjaia. https://elearning.univbejaia.dz/pluginfile.php/96365/mod_resource/content/0/colloques/cemaforad/pdf/delievre_bruno.pdf

Depover, C., Komis, V. et Karsenti, T. (2012). Le contrôle de qualité : un outil indispensable pour asseoir la légitimité de la formation à distance? Formation et profession, 20(2), 1-8.

Ehlers, U.-D. (2004). Quality in e-Learning from a Learner's Perspective. European Journal of Open, Distance and E-Learning.

Esfijani, A. (2018). Measuring quality in online education: A meta-synthesis. American Journal of Distance Education, $32(1), 57-73$.

Hew, K. F., Liu, S., Martinez, R., Bonk, C. et Lee, J. Y. (2004). Online education evaluation: What should we evaluate? In the Proceedings of the Association for Educational Communications and Technology (pp. 243-246). Chicago, IL: Association for Educational Communications and Technology. https://eric.ed.gov/?id=ED485142 
Pham, L., Limbu, Y. B., Bui, T. K., Nguyen, H. T. et Pham, H. T. (2019). Does e-learning service quality influence elearning student satisfaction and loyalty? Evidence from Vietnam. International Journal of Educational Technology in Higher Education, 16(7). https://link.springer.com/article/10.1186/s41239-019-0136-3

Savarieau, B. et Daguet, H. (2013). L'introduction des « classes virtuelles » synchrones, un moyen de renforcer la qualité de l'accompagnement en formation d'adultes? Frantice. net, 6, 107-118. 